\title{
Estudio serológico de la Tripanosomiasis Americana y factores asociados en perros de una comunidad rural de Yucatán, México
}

\author{
Serological study of Trypanosoma cruzi and associated factors in dogs from a rural \\ community of Yucatan, Mexico
}

\author{
JR Carrillo-Peraza ${ }^{\mathrm{a}, \mathrm{b}}$, P Manrique-Saide ${ }^{\mathrm{a}}$,JC Rodríguez-Buenfib ${ }^{\mathrm{b}}$, JF Escobedo-Ortegón ${ }^{\mathrm{c}}$, RI Rodríguez-Vivas ${ }^{\mathrm{b}}$ \\ ME Bolio-González ${ }^{b}$, M Barrera-Pérez ${ }^{c}$ E Reyes-Novelo ${ }^{c}$, CH Sauri-Arceo ${ }^{b}$

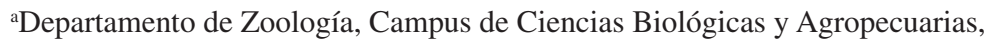 \\ Universidad Autónoma de Yucatán, Mérida, Yucatán, México.

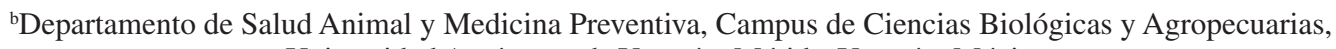 \\ Universidad Autónoma de Yucatán, Mérida, Yucatán, México. \\ ${ }^{c}$ Laboratorio de Zoonosis y Otras Enfermedades Transmitidas por Vectores, Centro de Investigaciones Regionales "Dr. Hideyo \\ Noguchi”, Universidad Autónoma de Yucatán, Mérida, Yucatán, México.
}

\begin{abstract}
SUMMARY
Chagas Disease is a protozoan infection caused by Trypanosoma cruzi that has spread to vertebrates, including humans. This parasite is prevalent in the canine population (Canis familiaris) of the State of Yucatan, Mexico. Few studies have reported the serological frequency and factors associated with exposure to T. cruzi of dogs populations in rural communities of Yucatan. This study aims to estimate the frequency and factors associated with anti-T. cruzi antibodies in dogs from a rural community in Yucatan. A research was carried out by convenience in 117 domestic dogs from the rural community. Blood samples from each dog to obtain serum were taken. The samples were analyzed by immunochromatography test for detecting anti-T. cruzi antibodies. A questionnaire to identify potential risk factors associated with seropositivity was administered to the owners of dogs. The response variables were analyzed by Chi square and variables with $\mathrm{P}<0.2$ were analyzed using logistic regression. Serological frequency of $29.9 \%$ (95\% CI: 21.61-38.21) was found. Factors associated with dogs with anti-T. cruzi were age ( $\operatorname{dogs}>1$ year old, $\mathrm{OR}=3.8 \mathrm{P}=0.02)$, place inhabited by the dog (dogs living outside the house, OR = 6.4 $\mathrm{P}=0.04)$ and presence of Triatoma dimidiata bugs in houses ( $\mathrm{OR}=4.7 \mathrm{P}=0.006)$. We conclude that dogs of the community under study present $T$. cruzi antibodies $(29.9 \%$ frequency) and three factors are associated with serologically positive dogs (adult dogs, dogs living outside the house and presence of bugs in the house).
\end{abstract}

Key words: Trypanosoma cruzi, dogs, risk factor, immunochromatographic.

\section{RESUMEN}

La Enfermedad de Chagas es producida por el protozoario Trypanosoma cruzi que causa una infección ampliamente diseminada en vertebrados, incluido el hombre. Escasas investigaciones han reportado la frecuencia serológica y los factores asociados a la exposición de $T$. cruzi en las poblaciones caninas de comunidades rurales de Yucatán. El presente estudio tiene por objetivo estimar la frecuencia serológica e identificar factores asociados a anticuerpos anti-T. cruzi en perros de una comunidad rural de Yucatán, México. Se realizó un estudio por conveniencia en 117 perros de la comunidad rural. Se tomaron muestras de sangre de cada perro para la obtención del suero. Las muestras fueron analizadas mediante una prueba de inmunocromatografía para detectar anticuerpos anti-T. cruzi. A los propietarios de los perros se les aplicó un cuestionario para identificar posibles factores de riesgos asociados a la seropositividad. Las variables de respuesta fueron analizadas mediante Chi cuadrado y las variables con $\mathrm{P}<0,2$ fueron analizadas mediante regresión logística. Se encontró una frecuencia serológica del 29,9\% (IC95\%: 21,61-38,21). Los factores asociados a perros con anticuerpos anti-T. cruzi fueron: edad (perros $>1$ año de edad, $\mathrm{OR}=3,8 \mathrm{P}=0,02$ ), lugar donde habitan los perro (perros en el peridomicilio, $\mathrm{OR}=6,4 \mathrm{P}=0,04$ ) y presencia de triatominos en las casas $(\mathrm{OR}=4,7 \mathrm{P}=0,006)$. Se concluye que los perros de la comunidad bajo estudio presentan anticuerpos anti-T. cruzi $(29,9 \%$ de frecuencia) y tres factores se asocian a perros serológicamente positivos (perros adultos, perros que viven en el peridomicilio y presencia de triatominos en la casa).

Palabras clave: Trypanosoma cruzi, perros, factores de riesgo, inmunocromatografía.

\section{INTRODUCCIÓN}

La Tripanosomiasis Americana (TA), también conocida como Enfermedad de Chagas, es producida por el protozoo flagelado, Trypanosoma cruzi (T. cruzi) y es

Aceptado: 04.07.2013.

* Km. 15.5 Carretera Mérida-Xmatkuil C.P. 9700; rbuenfil@uady.mx transmitida principalmente por triatominos hematófagos. La enfermedad se distribuye ampliamente desde el sur de Estados Unidos hasta Argentina. En México se estima que anualmente 7.700 individuos contraen la TA y en el 2006 se estimó que cerca de 1,1 millones de personas se encontraban infectados con T. cruzi (NTD 2006).

La TA se encuentra presente en el Estado de Yucatán (Zavala-Velázquez 2003) y Triatoma dimidiata (Latreille 
1811) (Hemiptera: Reduviidae) es el único vector reportado (Guzmán-Marín y col 1991, Dumonteil y col 2002). Este vector mantiene el ciclo selvático de la enfermedad alimentándose de animales silvestres (ratón y armadillo) y el ciclo doméstico/peridoméstico alimentándose de humanos y mamíferos domésticos/sinantrópicos (zarigüeya, gato y perro) (Zavala-Velázquez y col 1996, Ruíz-Piña y col 2002, Jiménez-Coello y col 2008). Los perros infectados suelen presentar signos clínicos tanto en la fase aguda como en la crónica. Sin embargo, la TA en perros es importante no solo debido a la enfermedad que causa sino porque juegan un papel importante en el ciclo de transmisión hacia los humanos (Campos-Valdéz y col 2001, Sosa-Jurado y col 2004, Estrada-Franco y col 2006). Los perros actúan como hospederos intradomiciliares y/o peridomiciliares (Estrada-Franco y col 2006). Diversos estudios reportan seroprevalencias del $24 \%$ al $65 \%$ en perros de diversos países de Latinoamérica (Estrada-Franco y col 2006, Gürtler y col 2007, Barbabosa-Pliego y col 2010). En Yucatán, México, los primeros reportes de perros infectados con T. cruzi fueron realizados por ZavalaVelázquez y col (1996). Posteriormente, Jiménez-Coello y col (2008) reportaron 17\% de seroprevalencia en muestras de perros callejeros y además 34\% de seroprevalencia en perros con propietario en áreas urbanas de Mérida, la ciudad capital de Yucatán, México (Jiménez-Coello y col 2010). Sin embargo, estudios sobre la exposición de los perros domésticos a T. cruzi en comunidades rurales de Yucatán son escasos. Las áreas rurales proveen las condiciones necesarias para que el vector manifieste sus habilidades para adaptarse a las casas (Briceño-León y col 2007). En las comunidades rurales de Yucatán, el vector puede encontrar un gran número de hospederos tanto silvestres como domésticos (Zavala-Velázquez y col 1996, Ruíz-Piña y col 2002), de los cuales puede alimentarse y transmitir el parásito. Además, en estas zonas el vector puede disponer de materiales para construir sus nidos y reproducirse (Menes y col 2006, Reyes-Novelo y col 2011). Junto a esto, se ha documentado que la mayoría de las comunidades de Yucatán tienen una sobrepoblación de perros (Ortega-Pacheco 2001) y como consecuencia una elevada relación persona/perro (1:1,7 a 1:4,6) (OrtegaPacheco y col 2007). Investigaciones han señalado como factor de riesgo el hecho de tener un mayor número de perros seropositivos en la casa (Estrada-Franco y col 2006).

Por tal motivo este estudio tiene por objetivo estimar la frecuencia serológica e identificar factores asociados a anticuerpos anti-T. cruzi en perros de una comunidad rural de Yucatán, México.

\section{MATERIAL Y MÉTODOS}

\section{SITIO DE ESTUDIO}

El estudio fue llevado al cabo en la comunidad rural de Molas localizada a $12 \mathrm{~km}$ al sur de la ciudad de
Mérida, Yucatán $\left(20^{\circ} 49^{\prime}\right.$ y $20^{\circ} 48^{\prime} \mathrm{N}$ y $89^{\circ} 37^{\prime}$ y $89^{\circ}$ $38^{\prime}$ O), con una población estimada de aproximadamente 2014 habitantes viviendo en 423 casas dentro de la reserva ecológica "Cuxtal" y rodeada por selva subtropical caducifolia ${ }^{1}$.

El clima de la región es cálido subhúmedo $\left(\mathrm{AW}_{0}\right)$, con temperatura anual promedio de $27-28^{\circ} \mathrm{C}$ y una precipitación pluvial de 800 a $1200 \mathrm{~mm}$ que ocurre principalmente durante la temporada de lluvias (mayo-octubre) ${ }^{2}$.

Se ha documentado que el $71 \%$ de las casas de Molas, Yucatán, tiene perros (Ortega-Pacheco y col 2007).

\section{POBLACIÓN DE ESTUDIO Y COLECTA DE MUESTRAS}

Se realizó un estudio por conveniencia en 117 perros de $>3$ meses de edad y de distintas razas. Los animales fueron estudiados entre noviembre 2010 y enero 2011 en toda la comunidad de Molas. Se tomaron muestras de sangre de cada perro mediante la punción de la vena cefálica. Las muestras de sangre completa fueron colectadas con jeringas y transferidas a tubos estériles, las cuales fueron llevadas al laboratorio para ser almacenadas a $4{ }^{\circ} \mathrm{C}$ por 5 horas y centrifugadas a $650 \mathrm{~g}$ por $10 \mathrm{minu}-$ tos; posteriormente el suero fue separado y conservado a $-20{ }^{\circ} \mathrm{C}$ hasta su análisis. Las muestras fueron tomadas bajo el mínimo estrés, con el consentimiento y la presencia del dueño de cada animal, siguiendo la normatividad vigente (NOM-062-ZOO-1999) para el manejo y la toma de muestras de animales en México.

\section{PRUEBA DE INMUNOCROMATOGRAFÍA}

Los sueros fueron analizados mediante la prueba rápida serológica Chagas Ab Rapid (Standard Diagnostics Bioline) de acuerdo a las instrucciones del fabricante. Esta prueba ha sido diseñada para la determinación cualitativa de anticuerpos contra T. cruzi (Cardinal y col 2006, Reithinger y col 2010, Rosypal y col 2010), presentando una sensibilidad de 99,2\% y una especificidad de $100 \%$ (Jung y col 2009). Esta prueba emplea una combinación de un anticuerpo específico unido a una proteína, la cual está conjugada con partículas de oro y un antígeno (H49, 1F8), unido a la membrana (Umezawa y col 1999). Como control positivo se utilizó el suero de un perro que mostró anticuerpos anti-IgG contra T. cruzi por medio de la prueba de ELISA y se determinó la presencia del agente por PCR. Como control negativo fue utilizado un suero de perro clínicamente sano y previamente negativo a anticuerpos (prueba de ELISA) y a la infección de T. cruzi (prueba de PCR).

\footnotetext{
www.merida.gob.mx/comisarias/, visitado el 12 de febrero 2013.
}

2 CONAGUA, Comisión Nacional del Agua. 2013. Boletín Meteorológico del Estado de Yucatán. www.conagua.gob.mx/OCPY07/Contenido/Documentos/BolEst.pdf.pdf, visitado el 12 de febrero 2013. 


\section{APLICACIÓN DE CUESTIONARIO}

Se aplicó un cuestionario a los propietarios de los perros, el cual fue completado por medio de sus respuestas y la observación directa del encuestador (Jiménez-Coello y col 2008, Jiménez-Coello y col 2010). El cuestionario se aplicó por cada perro para identificar los potenciales riesgos a la exposición con T. cruzi. La edad de los perros fue obtenida con base en la dentadura y la deposición de sarro sobre sus dientes (Ortega-Pacheco y col 2007); se consideró como cachorro al animal que tenía una edad de 3 meses a un año y adulto de 1 a 6 años. La ubicación del perro en la casa se consideró intradomiciliar si este habitaba dentro de la casa o peridomiciliar si deambulaba en el traspatio o alrededor de la casa. El lugar para dormir del perro se registró mediante la respuesta de los moradores y se categorizó como dentro o fuera de la casa. Otra variable estudiada fue si el perro se encontraba generalmente amarrado o suelto. La condición corporal se evaluó como mala (bajo de peso), regular o buena, usando el método descrito por Dorsten y Cooper (2004). La presencia/ausencia de aves y otros animales domésticos (cerdo, vaca, borrego, cabra o gato) se registró cuestionando a los propietarios si contaban con estos animales en su domicilio, además, se realizó una breve visita al traspatio de los moradores para confirmar la respuesta. Finalmente, se preguntó a los propietarios de los perros si habían visto insectos triatominos (vectores de T. cruzi) fuera o dentro de la casa. Se confió en las respuestas de los moradores, debido a que seis meses antes se realizó un estudio donde los propietarios de las casas participaron en la colecta de triatominos (Dumonteil y col 2009).

\section{ANÁLISIS ESTADÍSTICO}

La frecuencia serológica de perros seropositivos a T. cruzi fue calculada con base en los resultados de la prueba de inmunocromatografía y mediante la fórmula descrita por Thrusfield (2005). Para determinar los posibles factores asociados a la seropositividad de $T$. cruzi se realizó un análisis univariado mediante la prueba de Chi cuadrado. En este análisis se eliminaron aquellas variables que presentaban una $P>0,20$. A continuación, las variables restantes fueron analizadas por medio de una regresión logística binomial. La razón de momios ("Odds Ratio", OR) y los intervalos de confianza (IC) al 95\% fueron calculados mediante el uso del paquete de cómputo SPSS 15.0. (SPSS 2006) versión para Windows (SPSS Inc, Chicago IL, USA). Se consideró un nivel de significancia de $\mathrm{P}<0,05$.

\section{RESULTADOS}

En los perros estudiados se obtuvo una frecuencia de anticuerpos anti-T. cruzi de 29,9\% (IC95\%: 21,61$38,21)$. En el cuadro 1 se presentan los resultados de las variables exploradas mediante prueba de Chi cuadrado. Se observa que cuatro variables (edad, condición corporal, ubicación del perro en la casa y presencia de triatominos en la casa) tuvieron valores de $\mathrm{P}<0,20$, las cuales fueron sometidas al análisis de regresión logística. En el cuadro 2 se presenta los resultados del análisis de regresión logística donde se aprecia que las variables asociadas $(\mathrm{P}<0,05)$ a la presencia de perros con anticuerpos anti-T. cruzi fueron: edad (perros $>1$ año de edad, $\mathrm{OR}=$ 3,8 ), ubicación del perro en la casa (perros en el peridomicilio, $\mathrm{OR}=6,4$ ) y presencia de triatominos en la casa $(\mathrm{OR}=4,7)$.

\section{DISCUSIÓN}

La frecuencia de anticuerpos anti-T. cruzi obtenida mediante la prueba de inmunocromatografía en el presente estudio $(29,9 \%)$ indican una clara respuesta inmune de los perros debido a la exposición con el agente causante de la TA. Diferentes autores como Cardinal y col $\left(2006^{\text {b }}\right.$ y Rosypal y col (2011) han evaluado y reportado una elevada sensibilidad y especificidad en pruebas de inmunocromatografía en hospederos como el perro. Sin embargo, son pocos los estudios que utilizan este tipo de prueba por su reciente incursión en el mercado comercial (Rosypal y col 2010). A pesar de eso, si contrastamos los resultados del presente estudio con reportes que utilizan otras pruebas serológicas, se pueden encontrar similitudes con lo reportado por Montenegro y col (2002) quienes documentaron un $27,7 \%$ de seroprevalencia en perros de Costa Rica, Barbabosa-Pliego y col (2010) reportaron 24,5\% en perros del Estado de México y Jiménez-Coello y col (2010) estimaron un 34\% de seroprevalencia en perros de una zona urbana de Yucatán, México. Sin embargo, tanto en Sudamérica como Centroamérica se pueden encontrar frecuencias serológicas inferiores o superiores a lo encontrado en este estudio (Cardinal y col 2007, Gürtler y col 2007, Cardinal y col 2008, Romero-Peñuela y col 2008). Del mismo modo en México existe evidencia documentada de la presencia de anticuerpos contra $T$. cruzi en perros que van de 7,6\% hasta 65,4\% (Sosa-Jurado y col 2004, Estrada-Franco y col 2006, BarbabosaPliego y col 2010, Hernández y col 2010).

Los diferentes resultados en la frecuencia serológica de perros reportadas en América puede deberse principalmente a la diversidad y abundancia de las especies de vectores que habitan en cada región. La presencia de estos vectores de T. cruzi dependen de las condiciones ambientales de cada zona geográfica (temperatura, altitud), así como de las condiciones socioeconómicas de las viviendas en zonas rurales o urbanas (Sosa-Jurado y col 2004, Estrada-Franco y col 2006, Barbabosa-Pliego y col 2010). En zonas urbanas de Yucatán se han documentado en perros frecuencias de anticuerpos anti-T. cruzi que van del 14,4\% al 16,1\% (Zavala-Velázquez y col 1996, Jiménez-Coello y col 2008), con excepción de un reporte 
Cuadro 1. Análisis exploratorio de las variables asociadas con anticuerpos anti-Trypanososma cruzi mediante la prueba de Chi cuadrado en 117 perros de una comunidad rural de Yucatán, México. Yucatan, Mexico.

Exploratory analysis of the variables associated with anti-Trypanososma cruzi by Chi-square test on 117 dogs from a rural community in

\begin{tabular}{|c|c|c|c|c|c|}
\hline Variable & $\mathrm{N}$ & Positivo & Frecuencia (\%) & $\chi^{2}$ & Valor de $\mathrm{P}$ \\
\hline \multicolumn{6}{|l|}{ Sexo } \\
\hline Hembra & 40 & 13 & 32,5 & & \\
\hline Macho & 77 & 23 & 29,8 & 0,085 & 0,770 \\
\hline \multicolumn{6}{|l|}{ Edad } \\
\hline Cachorro (3 meses-1 año) & 35 & 5 & 14,2 & & \\
\hline Adulto (> 1-6 años) & 82 & 31 & 37,8 & 6,370 & $0,012 *$ \\
\hline \multicolumn{6}{|l|}{ Condición corporal } \\
\hline Buena & 47 & 19 & 40,7 & & \\
\hline Regular & 47 & 12 & 25,5 & & \\
\hline Mala & 23 & 5 & 21,7 & 3,543 & $0,170^{*}$ \\
\hline \multicolumn{6}{|l|}{ Ubicación del perro en la casa } \\
\hline Peridomicilio & 102 & 28 & 27,4 & & \\
\hline Intradomicilio & 15 & 8 & 53,3 & 4,112 & $0,043^{*}$ \\
\hline \multicolumn{6}{|c|}{ Como mantiene al perro en casa } \\
\hline Amarrado & 46 & 15 & 32,6 & & \\
\hline Suelto & 71 & 21 & 29,5 & 0,120 & 0,729 \\
\hline \multicolumn{6}{|l|}{ Lugar donde duerme } \\
\hline Fuera de la casa & 109 & 33 & 30,2 & & \\
\hline Dentro de la casa & 8 & 3 & 37,5 & 0,183 & 0,669 \\
\hline \multicolumn{6}{|l|}{ Presencia de aves } \\
\hline $\mathrm{Si}$ & 83 & 27 & 32,5 & & \\
\hline No & 34 & 9 & 26,4 & 0,416 & 0,519 \\
\hline \multicolumn{6}{|c|}{ Presencia de otros animales domésticos } \\
\hline $\mathrm{Si}$ & 63 & 17 & 26,9 & & \\
\hline No & 54 & 19 & 35,1 & 0,918 & 0,338 \\
\hline \multicolumn{6}{|c|}{ Presencia de triatominos en la casa } \\
\hline $\mathrm{Si}$ & 74 & 31 & 41,8 & & \\
\hline No & 43 & 5 & 11,6 & 11,694 & $0,001 *$ \\
\hline
\end{tabular}

Los valores marcados con $(*)$ son significativos $(\mathrm{P}<0,2)$.

del $34 \%$ en un estudio limitado a 35 perros (JiménezCoello y col 2010). Contrastados estos resultados con el presente estudio se puede notar que los valores encontrados en zonas urbanas son inferiores a lo reportado en este trabajo $(29,9 \%)$ realizado en una zona rural, esto se puede deber a que las casas de zonas rurales tienen un contacto más cercano con la selva, de donde posteriormente los triatominos infectados no domiciliados invaden los domicilios en ciertas épocas de año (Dumonteil y col 2002), alimentándose de animales domésticos como el perro (Guzmán-Marín y Col 1992, Gürtler y col 2007). Además, se han asociado dos factores que incrementan la afectación de la TA, las condiciones de las casas de las zonas rurales (Briceño-León y col 2007, OMS 2007) y la práctica común de producción de animales de traspatio (principalmente aves), los cuales son factores que ayudan al establecimiento del vector (Gürtler y col 2007).
La alta frecuencia de anticuerpos anti-T. cruzi en los perros estudiados puede deberse a los índices entomológicos reportados para el $T$. dimidiata en la región. Guzmán-Marín y col (1991) en una investigación llevada a cabo en 22 localidades de Yucatán, recolectaron T. dimidiata en el $61 \%$ de las casas estudiadas y un índice de infección con T. cruzi de $16 \%$, lo que podría explicar el probable contacto del vector con los perros.

En este estudio se identificaron tres factores asociados a perros con anticuerpos anti-T. cruzi. Los perros $>1$ años a 6 años de edad tuvieron mayor probabilidad de ser seropositivos $(\mathrm{OR}=3,8 \mathrm{P}=0,02)$ que los cachorros. Esto puede deberse a que los perros adultos tienen mayor tiempo de exposición al agente comparado con los cachorros (Rowland y col 2010). Esta asociación coincide con lo reportado por Estrada-Franco y col (2006) y Gürtler y col (2007) quienes han reportado que a mayor 
Cuadro 2. Resultados del análisis de la regresión logística binomial de las variables asociadas a serorreacción positiva a Trypanosoma cruzi en 117 perros de Molas, Yucatán, México. Yucatan, Mexico.

Results of logistic regression analysis of binomial variables associated with positive seroreaction Trypanosoma cruzi in 117 dogs Molas,

\begin{tabular}{|c|c|c|c|c|c|c|}
\hline Variables & $n$ & Positivo & Frecuencia $(\%)$ & $\mathrm{OR}^{*}$ & $\mathrm{IC}^{* *}$ & $\mathrm{P} * * *$ \\
\hline \multicolumn{7}{|l|}{ Edad } \\
\hline Cachorro (3 meses-1 año) & 35 & 5 & 14,2 & & & \\
\hline Adulto (> 1-6 años) & 82 & 31 & 37,8 & 3,8 & $1,16-12,90$ & 0,028 \\
\hline \multicolumn{7}{|c|}{ Ubicación del perro en la casa } \\
\hline Peridomicilio & 102 & 28 & 27,4 & & & \\
\hline Intradomicilio & 15 & 8 & 53,3 & 6,4 & $0,02-0,96$ & 0,045 \\
\hline \multicolumn{7}{|l|}{ Condición corporal } \\
\hline Buena & 47 & 19 & 40,7 & 0,7 & & 0,74 \\
\hline Regular & 47 & 12 & 25,5 & 1,27 & $0,47-3,45$ & 0,62 \\
\hline Mala & 23 & 5 & 21,7 & 1,61 & $0,45-5,73$ & 0,46 \\
\hline \multicolumn{7}{|c|}{ Presencia de triatomino en la casa } \\
\hline $\mathrm{Si}$ & 74 & 31 & 41,8 & & & \\
\hline No & 43 & 5 & 11,6 & 4,7 & $1,55-14,43$ & 0,006 \\
\hline
\end{tabular}

OR: *Razón de momios, IC: **Intervalo de confianza al 95\%, P: *** Estadísticamente significativo $(\mathrm{P}<0,05)$.

edad de los perros la frecuencia serológica a T. cruzi se incrementa.

De manera similar, los perros que se ubican en el peridomicilio de las casas tuvieron mayor probabilidad (OR $=6,4 \mathrm{P}=0,04)$ de ser positivos a anticuerpos anti-T. cruzi que los ubicados en el intradomicilio. En las comunidades rurales de Yucatán es común que los perros se ubiquen en el peridomicilio, lo que favorece que los animales sean picados por los triatominos infectados (Rowland y col 2010). Dumonteil y col (2007) señalan la importancia que tiene el peridomicilio como un área donde se mantienen las poblaciones de $T$. dimidiata provenientes de áreas selváticas. Junto a esto, diversos autores han documentado mayores colectas de $T$. dimidiata en el peridomicilio que dentro de la casa, lo cual es un factor que tiene como consecuencia mayores frecuencias serológicas en los animales que viven en esa área de la casa (Guzmán-Marín y col 1991, Guzmán-Marín y col 1992). También es común encontrar en estas comunidades rurales corrales para animales de producción (en uso o abandonados), que usan los triatominos como sitios de reproducción y refugio (WHO 2002, Zachary y col 2006).

Otro factor asociado a anticuerpos anti-T. cruzi en perros fue la presencia de triatominos en la casa $(\mathrm{OR}=$ 4,7 $\mathrm{P}=0,006)$. Diversas investigaciones han publicado la relación que existe entre la presencia de triatominos en las viviendas y la positividad de humanos y perros a anticuerpos anti-T. cruzi (Gürtler y col 1998). En otro estudio se ha relacionado los altos índices de infección de los triatominos con elevadas prevalencias de anticuerpos anti-T. cruzi en los perros (Diosque y col 2004). Por otro lado, se ha reportado que si se reduce el número de triatominos en las viviendas, las prevalencias de perros positivos a T. cruzi en perros también se reduce (Gürtler y col 1991, Cardinal y col 2006a ). Es indudable que la presencia de triatominos infectados en las casas es un factor de riesgo para los residentes, ya que personas y animales domésticos, como el perro, están en riesgo de adquirir la infección (Portugal-García y col 2011).

Se concluye que los perros de la comunidad rural bajo estudio presentan anticuerpos anti-T. cruzi $(29,9 \%$ de frecuencia) y tres factores se asocian a perros serológicamente positivos (perros adultos, perros que viven en el peridomicilio y la presencia de triatominos en la casa).

\section{AGRADECIMIENTOS}

Este estudio fue realizado bajo el auspicio del proyecto "Estudio multidisciplinario para la identificación de variables asociadas a la transmisión de enfermedades zoonóticas y enfermedades transmitidas por vector en Yucatán" (PROMEP 200810.5/12.58). Los autores pertenecen a la Red Epidemiología de Enfermedades Zoonóticas y Transmitidas por Vector de Importancia en Salud Pública.

\section{REFERENCIAS}

Barbabosa-Pliego A, P Campos-Gil, D Olivares-Hernández, J Aparicio-Burgos, R Montes de Oca-Jiménez, J Martínez-Castañeda, L Ochoa-García, C Guzman-Bracho, J Estrada-Franco, G Jain, CJ Vázquez. 2010. Prevalence of Trypanosoma cruzi in dogs (Canis familiaris) and triatomines during 2008 in a sanitary region of the State of Mexico, Mexico. Vector Borne Zoonotic Dis 10, 1-6.

Briceño-León RJ, G Méndez. 2007. The social determinants of Chagas disease and the transformations of Latin America. Mem Inst Oswaldo Cruz 102, 109-112. 
Campos-Valdéz G, R Barrera-Peña, R De la Luz Sánchez, E Jiménez-Cardoso, P Martínez-Labat. 2001. Anti-antibodies in dogs (Canis familiaris) from Tlalnepantla Municipality, State of Mexico. Bioquimia 26, 16-19.

Cardinal MV, MB Castañeda, MA Lauricella, MC Cecere, LA Ceballos, GM Vazquez-Prokopec, U Kitron, RE Gürtler. $2006^{\mathrm{a}}$. A prospective study of the effects of sustained vector surveillance following community-wide insecticide application on Trypanosoma cruzi infection of dogs and cats in rural northwestern Argentina. Am J Trop Med Hyg 75, 753-761.

Cardinal MV, R Reithinger, RE Gürtler. 2006 ${ }^{\mathrm{b}}$. Use of an immunochromatographic dipstick test for rapid detection of Trypanosoma cruzi in sera from animal reservoir hosts. $J$ Clin Microbiol 44, 3005-3007.

Cardinal MV, MA Lauricella, PL Marcet, MM Orozco, U Kitron, RE Gürtler. 2007. Impact of community-based vector control on house infestation and Trypanosoma cruzi infection in Triatoma infestans, dogs and cats in the Argentine Chaco. Acta Trop 103, 201-211.

Cardinal MV, MA Lauricella, LA Ceballos, L Lanati, PL Marcet, MJ Levin, U Kitron, RE Gürtler, AG Schijman. 2008. Molecular epidemiology of domestic and sylvatic Trypanosoma cruzi infection in rural northwestern Argentina. Int J Parasitol 38, 1533-1543.

Diosque P, A Marcelo-Padilla, R Oscar-Cimino, R MarinoCardozo, O Sanchez-Negrette, JD Marco, R Zacca, C Meza, A Juarez, H Rojo, R Rey, R Milagros-Corrales, JR Nasser, MA Basombrío. 2004. Chagas disease in rural areas of Chaco Province, Argentina: epidemiologic survey in humans, reservoirs, and vectors. Am J Trop Med Hyg 71, 590-593.

Dorsten CM, DM Cooper. 2004. Use of body condition scoring to manage body weight in dogs. J Am Assoc Lab Anim 43, 34-37.

Dumonteil E, S Gourbiére, M Barrera, E Rodríguez, H Ruiz, O Banos, J Ramírez, F Menu, J Rabinovich. 2002. Geographic distribution of Triatoma dimidiata and transmission dynamics of Trypanosoma cruzi in the Yucatan Peninsula of México. Am J Trop Med Hyg 67, 176-183.

Dumonteil E, F Tripet, MJ Ramírez-Sierra, V Payet, G Lanzaro, F Menu. 2007. Assessment of Triatoma dimidiata dispersal in the Yucatan peninsula of Mexico by morphometry and microsatellite markers. Am J Trop Med Hyg 76, 930-937.

Dumonteil E, MJ Ramirez-Sierra, J Ferral, M Euan-García, L Chávez-Núñez. 2009. Usefulness of community participation for the fine temporal monitoring of house infestation by non-domiciliated triatomines. J Parasitol 95, 469-71.

Estrada-Franco JE, V Bhatia, H Diaz-Albiter, L Ochoa-Garcia, A Barbabosa, JC Vázquez-Chagoyan, MA Martinez-Perez, C Guzman-Bracho, N Garg. 2006. Human Trypanosoma cruzi infection and seropositivity in dogs, Mexico. Emerg Infect Dis 12, 624-630.

Gürtler RE, MC Cecere, DN Rubel, RM Petersen, NJ Schweigmann, MA Lauricella, MA Buja, EL Segura, C Wisnivesky-Colli. 1991. Chagas disease in north-west Argentina: infected dogs as a risk factor for the domestic transmission of Trypanosoma cruzi. Trans $R$ Soc Trop Med Hyg 85, 741-745.

Gürtler RE, R Chuit, MC Cecere, MB Castañera, JE Cohen, EL Segura. 1998. Household prevalence of seropositivity from Trypanosoma cruzi in three rural villages in Northwest Argentina: environmental, demographic, and entomologic associations. Am J Trop Med 59, 741-749.

Gürtler RE, MC Cecere, MA Lauricella, MV Cardinal, U Kitron, JE Cohen. 2007. Domestic dogs and cats as sources of Trypanosoma cruzi infection in rural northwestern Argentina. Parasitology 134, 1-23.

Guzmán-Marín ES, MA Barrera-Pérez, ME Rodríguez-Félix, FJ Escobedo-Ortegón, JE Zavala-Velázquez. 1991. Índices entomológicos de Triatoma dimidiata en el Estado de Yucatán. Rev Biomed 2, 20-29.

Guzmán-Marín ES, MA Barrera-Pérez, ME Rodríguez-Félix, JE Zavala-Velázquez. 1992. Hábitos biológicos de Triatoma dimidiata en el Estado de Yucatán, México. Rev Biomed 3, 125-131.

Hernández GL, EA Rebollar-Téllez, F Infante, A Morón, A Castillo. 2010. Indicadores de Infestación, colonización e infección de Triatoma dimidiata (Latreille) (Hemiptera: Reduviidae) en Campeche, México. Neotrop Entomol 39, 1024-1031.

Jiménez-Coello M, M Poot-Cob, A Ortega-Pacheco, E Guzman-Marín, A Ramos-Ligonio, C Sauri-Arceo, K AcostaViana. 2008. American Trypanosomiasis in dogs from an urban and rural area of Yucatan, Mexico. Vector Borne Zoonotic Dis 8, 755-761.

Jiménez-Coello M, E Guzman-Marín, A Ortega-Pacheco, K Acosta-Viana. 2010. Serological survey of American Trypanosomiasis in dogs and their owners from an urban area of Mérida Yucatán, México. Transbound Emerg Dis 57, 33-36.

Jung JM, NJ Sang, CB Ki, CY Shink, KS Joo, YB Su. 2009. Evaluation of SD BIOLINE Chagas Ab Rapid Kit. Korean $J$ Lab Med 29, 48-52.

Menes HM, EM Monroy, MD Bustamante, B Moguel, RA Rodas, E Solórzano, M García. 2006. Estudio de las preferencias de hábitat no domiciliar del principal vector de la Enfermedad de Chagas en Guatemala, Triatoma dimidia$t a$, y sus implicaciones para el control vectorial. Universidad de San Carlos de Guatemala Dirección General de Investigación Programa Universitario de Investigación en Ciencia Básica. Diciembre de 2006. Guatemala.

Montenegro VM, M Jiménez, JC Pinto-Dias, R Zeledón. 2002. Chagas disease in dogs from endemic areas of Costa Rica. Mem Inst Oswaldo Cruz 97, 491-494.

NTD, Department of Control of Neglected Tropical Diseases. 2006. Innovative and Intensified Disease Management (IDM). WHO/NTD/IDM. Estimación cuantitativa de la enfermedad de Chagas en las Américas. Docto. OPS/ HDM/CD/425-06.

OMS, Organización Mundial de la Salud. Reporte sobre la enfermedad de Chagas. 2007. Noticias TDR. 17-20 de abril de 2005, actualizado en julio de 2007, Buenos Aires, Argentina.

Ortega-Pacheco A. 2001. La sobrepoblación canina: un problema con repercusiones potenciales para la salud humana. Rev Biomed 12, 290-291.

Ortega-Pacheco A, JC Rodríguez-Buenfil, ME Bolio-González, CH Sauri-Arceo, M Jiménez-Coello, CL Forsberg. 2007. A survey of dog population in urban and rural areas. Anthrozoos 20, 261-274.

Portugal-García C, Z García-Vázquez, V Monteón-Padilla, V 
Chávez-López, M Olamendi-Portugal, C Ramos. 2011. Anticuerpos contra Trypanosoma cruzi en humanos y perros y presencia del parásito en Meccus pallidipennis en la localidad de Puente Pantitlán, Morelos, México. Rev Biomed 22, 67-75.

Reithinger R, MJ Grijalva, RF Chiriboga, B Alarcón De Noya, JR Torres, N Norma-Pavia, P Manrique-Saide, MV Cardinal, RE Gürtler. 2010. Rapid detection of Trypanosoma cruzi in humans serum by use of an inmunochromatographic dipstick test. J Clin Microbiol 48, 3003-3007.

Reyes-Novelo E, H Ruíz-Piña, J Escobedo-Ortegón, M Barrera-Pérez. 2011. Biología y ecología de Triatoma dimidiata (Latreille, 1811), algunos aspectos de estudio. Dugesiana $18,11-16$.

Romero-Peñuela M, J Sánchez-Valencia. 2008. Seroprevalencia de Trypanosoma cruzi por la técnica de Western Blot en población canina del Departamento del Tolima, Colombia. Revista veterinaria y zootecnia 3, 48-52.

Rosypal AC, R Hill, S Lewis, K Braxton, AM Zajac, DS Lindsay. 2010. Toxoplasma gondii and Trypanosoma cruzi antibodies in dogs from Virginia. Zoonoses Public Hlth 57, 76-80.

Rosypal AC, R Hill, S Lewis, SC Barr, S Valadas, SM Gennari, DS Lindsay. 2011. Evaluation of a rapid immunochromatographic dipstick test for detection of antibodies to Trypanosoma cruzi in dogs experimentally infected with isolates obtained from Opossums (Didelphis virginiana), Armadillos (Dasypus novemcinctus), and dogs (Canis familiaris) from the United States. J Parasitol 97, 140-143.

Rowland ME, J Maloney, S Cohen, MJ Yabsley, J Huang, M Kranz, A Green, JR Dunn, LR Carpenter, TF Jones, AC Moncayo. 2010. Factors associated with Trypanosoma cruzi exposure among domestic canines in Tennessee. $J$ Parasitol 96, 547-551.
Ruíz-Piña H, A Cruz-Reyes. 2002. The opossum Didelphis virginiana as a synanthropic reservoir of Trypanosoma cruzi in Dzidzilché, Yucatán, México. Mem Inst Oswaldo Cruz. 97, 613-620.

Sosa-Jurado F, J Zumaquero-Ríos, R Reyes, A Cruz-García, C Guzmán-Bracho, V Monteón. 2004. Factores bióticos y abióticos que determinan la seroprevalencia de anticuerpos contra Trypanosoma cruzi en el municipio de Palmar de Bravo, Puebla, México. Salud Pública Mex 46, 39-48.

SPSS. 2006. SPSS 15.0. SPSS Inc., 233 South Wacker Drive, $11^{\text {th }}$ Floor Chicago, IL, USA.

Thursfield M. 2005. Veterinary Epidemiology. $3^{\text {rd }}$ ed.. Blackwell Science, London, UK, Pp 53-54.

Umezawa ES, SF Bastos, ME Camargo, LM Yamauchi, M Santos, A Gonzalez, B Zingales, MJ Levin, O Sousa, R Rangel-Aldao, JF Da Silveira. 1999. Evaluation of Recombinant Antigens for Serodiagnosis of Chagas' Disease in South and Central America. J Clin Microbiol 37, 1554-1560.

WHO, World Health Organization. 2002. Control of Chagas disease: second report of the WHO expert committee. Technical reports series 905. Geneva: The WHO, Switzerland.

Zachary ML, NM Bowman, V Kawai, LA Waller, JG Cornejodel Carpio, EB Cordova, RH Gilma, C Bern. 2006. Periurban Trypanosoma cruzi- infected Triatoma infestans, Arequipa, Peru. Emerg Infect Dis 12, 1345-1352.

Zavala-Velázquez J, M Barrera-Pérez, ME Rodríguez-Félix, E Guzmán-Marín, H Ruíz-Piña. 1996. Infection by Trypanosoma cruzi in mammals in Yucatan, Mexico: A serological and parasitological study. Rev Inst Med Trop São Paulo 38, 289-292.

Zavala-Velázquez J. 2003. La enfermedad de Chagas en el Estado de Yucatán, México (1940-2002). Rev Biomed 14, 35-43. 
